\title{
Association of dairy intake with all-cause, cancer, and cardiovascular disease mortality in Japanese adults: a 25-year population-based cohort
}

\author{
Yukai Lu $^{1} \cdot$ Yumi Sugawara$^{1}\left[\right.$ D Sanae Matsuyama ${ }^{1} \cdot$ Akira Fukao $^{2} \cdot$ Ichiro Tsuji $^{1}$
}

Received: 3 June 2021 / Accepted: 29 October 2021 / Published online: 8 November 2021

(c) The Author(s) 2021

\begin{abstract}
Purpose The association between dairy intake and mortality remains uncertain, and evidence for the Japanese population is scarce. We aimed to investigate the association between dairy intake and all-cause, cancer, and cardiovascular disease (CVD) mortality in Japanese adults.

Methods A total of 34,161 participants (16,565 men and 17,596 women) aged 40-64 years without a history of cancer, myocardial infarction, or stroke at baseline were included in the analysis, using data from the Miyagi Cohort Study initiated in 1990. Milk, yogurt, and cheese intake were obtained using a validated food frequency questionnaire. Total dairy intake was calculated as the sum of milk, yogurt, and cheese intake and then categorized by quartile. The outcomes were all-cause, cancer, and CVD mortality. Cox proportional hazards regression models were used to estimate multivariable hazard ratios (HRs) and 95\% confidence intervals (CIs) for mortality risks.

Results During 750,016 person-years of follow-up, the total number of deaths was 6498, including 2552 deaths due to cancer and 1693 deaths due to CVD. There was no association between total dairy intake and all-cause, cancer, and CVD mortality for both men and women. We also examined the associations between subgroup dairy products and mortality. For milk and yogurt intake, our results suggest null associations. However, cheese intake was modestly associated with lower all-cause mortality in women; compared with non-consumers, the multivariable HRs (95\%CIs) were 0.89 (0.81-0.98) for 1-2 times/ month, $0.88(0.78-1.00)$ for 1-2 times/week, and 0.89 (0.74-1.07) for 3 times/week or almost daily $(p$ trend $=0.016)$.

Conclusion Dairy intake was not associated with mortality in Japanese adults, except for limited evidence showing a modest association between cheese intake and a lower all-cause mortality risk in women.
\end{abstract}

Keywords Dairy $\cdot$ Milk $\cdot$ Yogurt $\cdot$ Cheese $\cdot$ Cancer $\cdot$ Cardiovascular disease $\cdot$ Mortality

$\begin{array}{ll}\text { Abbreviations } \\ \text { ANOVA } & \text { Analysis of variance } \\ \text { CHD } & \text { Coronary heart disease } \\ \text { CI } & \text { Confidence interval } \\ \text { CVD } & \text { Cardiovascular disease } \\ \text { FFQ } & \text { Food frequency questionnaire } \\ \text { HR } & \text { Hazard ratio } \\ \text { MI } & \text { Myocardial infarction }\end{array}$

Yumi Sugawara

yumi1717@med.tohoku.ac.jp

1 Division of Epidemiology, Department of Health Informatics and Public Health, Graduate School of Medicine, Tohoku University School of Public Health, 2-1 Seiryo-machi, Aoba-ku, Sendai 980-8575, Japan

2 Miyagi Cancer Society, Sendai, Japan

\section{Introduction}

Dairy products contribute various valuable nutrients to the overall diet, including protein, vitamins, and minerals, and consumption of dairy products is recommended in most dietary guidelines worldwide [1]. Previous studies have suggested that associations between dairy intake and multiple health outcomes, including diabetes mellitus [2], cardiovascular diseases (CVD) [3], breast cancer [4, 5], and colorectal cancer [5, 6], are null or weak inverse. Minerals in milk such as calcium, potassium, and magnesium may have played a role in the effect of milk on reducing blood pressure, which then may contribute to lowering the risk of CVDs [7]. However, recent randomized controlled trials have shown dairyrich diet has no effect on blood pressure compared to dairyfree diet $[8,9]$. Also, dairy products have a high content of saturated fat which raises low-density lipoprotein cholesterol 
level, consequently contributing to higher risk of CVDs [10], but current evidence has shown that neither whole milk nor low-fat milk has been clearly associated with CVDs [11]. Moreover, calcium in milk is related to protecting against the breast cancer and colorectal cancer risks [5].

However, it is possible that high consumption of dairy foods is associated with increased risks of prostate cancer $[5,12]$. Greater concentrations of insulin-like growth factor I (IGF-I) has been associated with the elevated prostate cancer risk, and milk consumption may increase IGF-I blood concentrations [13]. Evidence also has showed that total dairy intake is associated with a higher risk of endometrial cancer, particularly among postmenopausal women who are not currently using hormone therapy [14], which may attribute to the sex-hormone content of dairy products such as estrogen [15]. Therefore, whether dairy intake is beneficial or harmful to health is controversial due to the various nutrients in dairy products.

Numerous meta-analyses have investigated the association between dairy intake and mortality, but their results have been controversial [16-24]. Some suggested that higher dairy intake was associated with a lower mortality risk [16, 23], whereas others suggested a null association [17-19, 21, $22,24]$. Larsson et al. argued that it was perhaps inappropriate to pool the results due to their considerable heterogeneity [20]. It should be mentioned that most studies are conducted in Europe or North America where dairy products are traditionally consumed far more than in other regions [16]. Several cohort studies from Japan examining dairy intake and mortality showed inconsistent results [25-27]. One study found that milk drinking was associated with a lower risk of CVD mortality and cancer mortality in men [27], but the other two suggested null associations [25, 26]. Thus, whether different patterns of dairy intake between Western and Asian populations may be associated with mortality differently has been unclear. It also needs to be noted that different kinds of dairy products vary in their nutrient composition, so they may have different effects on health [16]. For example, previous studies suggested that yogurt or cheese rather than milk intake was associated with a lower risk of all-cause mortality [16, 28, 29].

Thus, the aim of the present study was to examine the association of dairy intake with all-cause, cancer, and CVD mortality in Japanese adults, using a large-scale populationbased cohort with follow-up over 25 years.

\section{Materials and methods}

\section{Study participants}

The data used in the present study came from the Miyagi Cohort, the design of which has been described in detail elsewhere [30, 31]. In brief, between June and August 1990, a self-administered questionnaire on various health conditions was delivered to all residents aged 40-64 years $(n=51,921)$ in 14 municipalities of Miyagi Prefecture, northeastern Japan. Of them, 47,605 were confirmed to be eligible (response rate: 91.7\%) (Fig. 1).

For the present analysis, one participant withdrawn from the study before follow-up starting, 2137 participants who had a history of cancer, myocardial infarction, or stroke at baseline, and 11,306 persons who did not answer the questions on milk, yogurt, or cheese intake were excluded. Eventually, 34,161 responses (16,565 men and 17,596 women) were included for the present analysis.

The study protocol was approved by the institutional review board of the Tohoku University School of Medicine (Approval No. 2014-1-838). We considered the return of self-administered questionnaires signed by the participants to imply their consent to participate in the study.

\section{Dietary assessment and dairy intake (exposure)}

Participants were asked about the average intake of dairy products including milk, yogurt, and cheese, as well as other food items, during the previous year, using a validated food frequency questionnaire (FFQ). The FFQ included 39 food

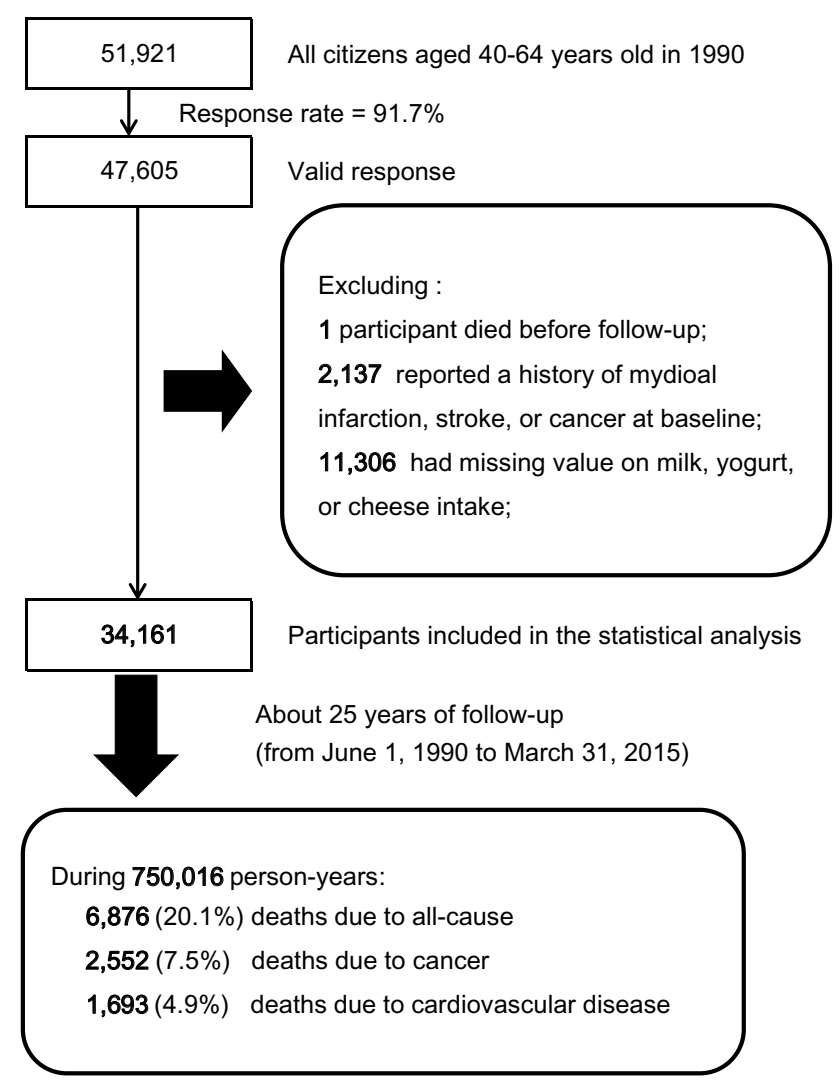

Fig. 1 Flowchart of study participants 
items and several beverages. For dairy products intake, participants were required to choose from the following five categories: "almost never", "1-2 times/month", "1-2 times/ week", "3-4 times/week", and "almost daily".

We also conducted a validation study for the FFQ we used for this study previously [32]. The age- and total energyadjusted Spearman's correlation coefficients between 3-day diet records and the FFQ were 0.72 for milk, 0.56 for yogurt, and 0.36 for cheese in men, and 0.65 for milk, 0.60 for yogurt, and 0.36 for cheese in women.

The volume of each food intake was calculated by converting the intake frequency from the FFQ into a daily intake volume (g/day). Daily intake was calculated by multiplying the average number of daily servings (times/day) by an assigned portion size (g/time) from the FFQ based on the median values observed in the validation study. Total dairy intake was calculated as the sum of daily intake of milk, yogurt, and cheese and was then sex-specifically categorized by quartile, with Q1 the lowest quartile and Q4 the highest one. For estimation of energy and other nutrient intakes from the food intake volume based on the FFQ, a food composition table that corresponded to the items listed in the questionnaire was used. This food composition table was developed using the Standard Tables of Food Composition published by the Science and Technology Agency of Japan [33].

\section{Follow-up}

The primary outcomes were all-cause, cancer, and CVD mortality, and the secondary outcomes were coronary heart disease (CHD) and stroke morality as well as lung cancer, gastric cancer, and colorectal cancer mortality. To follow-up the participants for mortality and migration, we established a Follow-up Committee [30, 34, 35], consisting of the Miyagi Cancer Society, the Community Health Divisions of all 14 municipalities, the Department of Health and Welfare of Miyagi Prefectural Government, and the Division of Epidemiology, Tohoku University Graduate School of Medicine. The Committee periodically reviewed the Residential Registration Record of each municipality. With this review, we identified participants who had either died or emigrated during the follow-up period. We discontinued follow-up with those who had emigrated from the study area, because the Committee could not review the Residential Registration Record from outside the study area.

For identified decedents, we further investigated cause of death by reviewing the death certificates of the participants at Public Health Centres in the study area. The cause of death was defined according to the International Classification of Diseases (ICD) 9th revision (ICD-9) between June 1, 1990 and December 31, 1998 and the 10th revision (ICD-10) between January 1, 1999 and March 31,
2015. Death due to CVD was coded by ICD-9:390-459 or ICD-10:I00-I99 (CHD: ICD-9:410-414 or ICD-10:I20I25; stroke:ICD-9:430-438 or ICD-10:I60-I69), and death due to cancer was coded by ICD-9:140-239 or ICD-10:C00-D09 (lung cancer:ICD-9:162 or ICD-10:C34; gastric cancer:ICD-9:151 or ICD-10:C16; colorectal cancer:ICD-9:153-154 or ICD-10:C18-C20).

Participants were followed up from June 1, 1990 to March 31,2015 . The number of person-years of follow-up for each participant was counted from the beginning of follow-up until the date of death, the date of emigration from the study districts, or the end of follow-up, whichever occurred first. During the follow-up period, 2997 participants (8.8\%) were lost to follow-up.

\section{Statistical analysis}

Cox proportional hazards model was used to calculate the sex-specific hazard ratios (HRs) and 95\% confidence intervals (95\% CIs) for mortality according to the quartile of total dairy intake, with participants in the lowest quartile (Q1) as the reference. Dummy variables were created for each group of exposure and categorical covariates. Missing values of each covariate were classified into an extra group. Time of follow-up was used as the time scale. Multivariable models were adjusted as follows: Model 1 was adjusted for age (continuous); Model 2 was further adjusted for education level (junior high school or lower, high school, college or higher, or missing), BMI ( $<18.5 \mathrm{~kg} / \mathrm{m}^{2}, 18.5-24.9 \mathrm{~kg} / \mathrm{m}^{2}, \geq 25.0 \mathrm{~kg} /$ $\mathrm{m}^{2}$, or missing), smoking status (never, former, $<20$ cigarettes/day, $\geq 20$ cigarettes/day, or missing), alcohol drinking status (never, former, current, or missing), and history of disease [hypertension and diabetes mellitus (yes or no for each)]; Model 3 was further adjusted for energy intake (in tertiles, or missing), vegetable and fruit intake (in tertiles, or missing), and fish intake (in tertiles, or missing). In addition, a test for trend was also conducted by coding the exposure variable using the median value of each category in the models.

A sensitivity analysis was also conducted by excluding deaths in the initial three years of follow-up, considering the possible reverse causality where health condition at baseline may affect dairy intake. Then, several stratified analyses according to age ( $<50$ vs. $\geq 50 \mathrm{y}), \mathrm{BMI}(<25.0$ vs. $\geq 25.0 \mathrm{~kg} / \mathrm{m}^{2}$ ), and smoking status (current vs. non-current) were also conducted, because these covariates which have a great impact on health outcomes may differ the association between dairy intake and mortality. A test for interaction was also performed by adding an additional crossproduct term of exposure variable and stratified covariate to the models. Because 11,306 persons with missing data on milk, yogurt, or cheese were excluded from the present analysis, which may affect the results, multiple imputations 
for missing data on dairy products were also applied. Five datasets with missing values being imputed according to age, sex, and other covariates, and Cox models were then created and applied to calculate the pooled HRs and 95\% CIs for mortality using the five imputed datasets [36].

Milk, yogurt, and cheese intake frequency were categorized into four groups based on FFQ responses. To obtain sufficient participants in each group, we combined "almost never" and "1-2 times/month" for milk, and "3-4 times/ week" and "almost daily" for yogurt and cheese. Butter was not included because it is distinct from other dairy foods in nutritional components and its correlation between 3-day diet records and the FFQ ( 0.20 for men and 0.11 for women) was much lower than other dairy products. All analyses were repeated using each dairy product intake frequency as the exposure variable, with the least frequent group as the reference.

All analyses were performed using SAS version 9.4 (SAS Inc., Cary, NC, USA). All statistical tests described were two-sided, and differences at $p<0.05$ were considered statistically significant.

\section{Results}

\section{Baseline characteristics}

During 750,016 person-years of follow-up, the total number of deaths was 6876 (4354 men and 2522 women), including 2552 deaths due to cancer (1713 men and 839 women) and 1693 deaths due to CVD (1048 men and 645 women). Table 1 shows the baseline characteristics by total dairy intake. The mean (standard deviation) total dairy intake was 125.9 (93.9) g/day for men and 148.1 (94.9) g/day for women, which was similar to that of general Japanese population (mean $130.1 \mathrm{~g} / \mathrm{day}$ ) [37] but was less than half of the amount in western countries measured in previous studies [38-42]. In both men and women, people with higher dairy intake were more likely to have a high education level, to be never smokers, to have high energy intake, or to have high vegetable and fruit intake.

\section{Dairy intake and mortality}

The sex-specific associations between total dairy intake and all-cause, cancer, and CVD mortality are presented in Table 2. However, there were no associations between total dairy intake and all-cause, cancer, and CVD mortality in both men and women. Tables 3, 4 and 5 show the associations between milk, yogurt, and cheese intakes and mortality, respectively. Similarly, our results suggest null associations between milk or yogurt intake and all-cause, cancer, and CVD mortality in both men and women (Tables 3,4).
However, for cheese intake (Table 5), a modest association with a lower risk of all-cause mortality was observed in women; compared with non-consumers, the multivariable HRs $(95 \% \mathrm{CI})$ were $0.89(0.81-0.98)$ for $1-2$ times/month, $0.88(0.78-1.00)$ for $1-2$ times/week, and $0.89(0.74-1.07)$ for 3 times/week or almost daily ( $p$ trend $=0.016$ ). We also examined the association between dairy intake and secondary outcomes including CHD and stroke mortality, as well as lung cancer, gastric cancer, and colorectal cancer mortality, but no association was found (e-Tables $1 \& 2$ ).

\section{Sensitivity analyses}

We conducted a sensitivity analysis by excluding deaths in the initial three years of follow-up, but the results were not essentially changed for total dairy intake and subgroup dairy products (e-Tables 3 and 4). We also conducted stratified analyses by age, BMI, and smoking status (e-Tables 5-8), and the results did not differ by those stratified variables. Moreover, we applied multiple imputation for missing values for dairy products and re-analyzed the imputed data, but the pattern of observed results remained for both total dairy intake and subgroup dairy products (e-Tables 9 and 10).

\section{Discussion}

\section{Main findings}

The present study examined the associations of both total dairy intake and subgroup dairy products with all-cause, cancer, and CVD mortality using a large-scale cohort study of the Japanese population with a follow-up period up to 25 years. The results suggested that dairy intake was not associated with mortality, except for limited evidence showing a modest association between cheese intake and a lower risk of all-cause mortality in Japanese women.

\section{Comparisons with previous studies}

There were no associations between total dairy intake and all-cause, cancer, and CVD mortality, which were in line with previous meta-analyses [16, 18, 24]. One suggested that total dairy products intake (per $200 \mathrm{~g} /$ day) was not associated with all-cancer mortality risk (RR: 0.99, 95\% CI 0.96-1.03), but there was considerable heterogeneity $\left(I^{2}=62.2 \%, p=0.005\right)$ [18]. The other study found that the highest group of total dairy intake was not associated with cancer mortality (RR: 0.99, 95\% CI 0.92-1.07) compared to the lowest group [24]. Most cohort studies included in the meta-analyses were from Western populations, and no meta-analyses reported results stratified by study regions. We identified several cohort studies from Asia [26, 43-45]. 
Table 1 Characteristics at baseline according to total dairy intake $(n=34,161)$

\begin{tabular}{|c|c|c|c|c|}
\hline & \multicolumn{4}{|c|}{ Quartile of total dairy intake ${ }^{\beta}$} \\
\hline & Q1 & Q2 & Q3 & Q4 \\
\hline \multicolumn{5}{|l|}{$\operatorname{Men}(n=16,565)$} \\
\hline No. of participants & 3979 & 4195 & 3366 & 5025 \\
\hline Dairy intake $(\mathrm{g} / \text { day })^{\alpha}$ & $6.6(7.5)$ & $71.5(27.7)$ & $180.0(41.8)$ & $229.7(26.7)$ \\
\hline Range of quartiles (g/day) & $0-40.8$ & $44.9-109.8$ & $110.8-210.0$ & $211.0-325.0$ \\
\hline Age $(\text { years })^{\alpha}$ & $50.1(7.4)$ & $49.7(7.3)$ & $51.2(7.5)$ & $50.7(7.4)$ \\
\hline College or higher $(\%)^{\beta}$ & 12.6 & 14.8 & 16.1 & 19.3 \\
\hline $\operatorname{BMI}\left(\mathrm{kg} / \mathrm{m}^{2}\right)^{\alpha}$ & $23.5(2.8)$ & $23.7(2.8)$ & $23.7(2.8)$ & $23.5(2.6)$ \\
\hline Never smokers (\%) & 14.8 & 18.0 & 20.1 & 23.3 \\
\hline Never alcohol drinkers $(\%)$ & 14.3 & 13.6 & 15.9 & 16.6 \\
\hline Time spent walking (> 1 h/day) (\%) & 44.5 & 42.2 & 44.5 & 42.5 \\
\hline Energy intake $(\mathrm{kJ} / \text { day })^{\alpha}$ & $7126.9(2545.4)$ & $7496.3(2443.0)$ & $7742.4(2386.4)$ & $8050.9(2376.2)$ \\
\hline Fish intake $(\mathrm{g} / \text { day })^{\alpha}$ & $56.7(35.3)$ & $58.7(33.5)$ & $63.3(34.7)$ & $63.0(34.2)$ \\
\hline Vegetable and fruit intake $(\mathrm{g} / \mathrm{day})^{\alpha}$ & $146.8(101.1)$ & $165.8(100.9)$ & $188.4(110.0)$ & $216.9(112.9)$ \\
\hline History of hypertension (\%) & 17.1 & 17.5 & 17.2 & 16.8 \\
\hline History of diabetes $(\%)$ & 3.5 & 4.2 & 6.5 & 5.9 \\
\hline \multicolumn{5}{|l|}{ Women $(n=17,596)$} \\
\hline No. of participants & 4396 & 4135 & 4514 & 4551 \\
\hline Dairy intake $(\mathrm{g} / \text { day })^{\alpha}$ & $17.5(19.0)$ & $103.8(32.1)$ & $212.9(2.6)$ & $250.1(27.0)$ \\
\hline Range of quartiles (g/day) & $0-49.4$ & $49.9-205.0$ & $210.0-224.5$ & $229.3-310.0$ \\
\hline Age $(\text { years })^{\alpha}$ & $51.1(7.4)$ & $49.8(7.1)$ & $52.2(7.2)$ & $50.6(7.3)$ \\
\hline College or higher $(\%)^{\beta}$ & 9.6 & 13.8 & 14.4 & 19.1 \\
\hline $\operatorname{BMI}\left(\mathrm{kg} / \mathrm{m}^{2}\right)^{\alpha}$ & $23.8(3.3)$ & $23.6(3.1)$ & $23.7(3.0)$ & $23.5(3.0)$ \\
\hline Never smokers $(\%)$ & 86.3 & 89.8 & 90.7 & 92.2 \\
\hline Never alcohol drinkers (\%) & 69.6 & 69.3 & 71.6 & 68.9 \\
\hline Time spent walking (> 1 h/day) (\%) & 44.0 & 44.2 & 45.1 & 42.2 \\
\hline Energy intake $(\mathrm{kJ} / \mathrm{day})^{\alpha}$ & $5108.0(1390.5)$ & $5528.2(1321.6)$ & $5819.0(1353.1)$ & $6050.7(1292.3)$ \\
\hline Fish intake $(\mathrm{g} / \text { day })^{\alpha}$ & $49.6(29.5)$ & $53.8(28.6)$ & $56.3(29.2)$ & $59.1(28.7)$ \\
\hline Vegetable and fruit intake $(\mathrm{g} / \text { day })^{\alpha}$ & $213.7(116.9)$ & $246.4(111.4)$ & $252.5(112.8)$ & $291.7(112.9)$ \\
\hline History of hypertension (\%) & 20.8 & 17.1 & 19.1 & 17.8 \\
\hline History of diabetes (\%) & 2.2 & 1.8 & 3.8 & 3.1 \\
\hline
\end{tabular}

${ }^{\alpha}$ Mean (standard deviation) for all such values

${ }^{\beta}$ Aged $\geq 19$ y when participants had completed their education

One study from Japan showed that consumption of milk and dairy products (per $100 \mathrm{~g} /$ day) was inversely associated with CVD mortality risk in women (HR: $0.86,95 \%$ CI 0.74-0.99), but not in men [26]. Studies from Taiwan ( 0 vs. $>7$ times/week) and Iran (per serving/day; $230 \mathrm{~g}$ for milk and yogurt and $28 \mathrm{~g}$ for cheese) both suggested that total dairy intake was inversely associated with allcause and CVD mortality, but not cancer mortality [43, 44], and the Iranian study also found that the association was more apparent for low-fat dairy intake [44]. Another study from Singapore suggested a marginally significant inverse association between total dairy intake and CVD mortality, especially stroke mortality, but only in men and those without a prior history of CVD [45].
Milk intake was not associated with all-cause, cancer, and CVD mortality in the present study. Numerous metaanalyses generally reported null associations [16, 18, 19, 21, 22, 24], but studies conducted in Asian populations showed controversial results [27, 44, 46, 47]. For all-cause mortality, four meta-analyses found no association with milk intake $[18,19,21,22]$, although three of them had considerable heterogeneity $\left(I^{2}: 72.3-97.4 \%\right)[18,19,22]$. Two cohort studies from Iran and China suggested no association between milk and all-cause mortality [44, 46], whereas one Japanese study found that milk intake was associated with a lower risk only in men aged 65 years or older, but not in women [27]. An Iranian study suggested that consuming whole milk daily or more was associated with a higher risk 
Table 2 Association between total dairy intake and mortality $(n=34,161)^{\alpha}$

\begin{tabular}{|c|c|c|c|c|c|}
\hline & \multicolumn{4}{|c|}{ Quartile of total dairy intake ${ }^{\beta}$} & \multirow[t]{2}{*}{$P$ trend $^{\gamma}$} \\
\hline & Q1 & Q2 & Q3 & Q4 & \\
\hline \multicolumn{6}{|l|}{ Men } \\
\hline Person-years & 84,007 & 89,576 & 71,387 & $1,08,493$ & \\
\hline \multicolumn{6}{|c|}{ All-cause mortality } \\
\hline No. of death & 1102 & 1023 & 956 & 1273 & \\
\hline Model $1^{\delta}$ & 1.00 (ref.) & $0.91(0.84-0.99)$ & $0.95(0.87-1.03)$ & $0.84(0.78-0.92)$ & 0.003 \\
\hline $\operatorname{Model} 2^{\varepsilon}$ & 1.00 (ref.) & $0.94(0.86-1.02)$ & $0.98(0.90-1.07)$ & $0.91(0.84-0.99)$ & 0.174 \\
\hline Model $3^{\zeta}$ & 1.00 (ref.) & $0.94(0.87-1.03)$ & $0.98(0.90-1.07)$ & $0.93(0.85-1.01)$ & 0.328 \\
\hline \multicolumn{6}{|c|}{ Cancer mortality } \\
\hline No. of death & 437 & 421 & 363 & 492 & \\
\hline Model $1^{\delta}$ & 1.00 (ref.) & $0.95(0.83-1.08)$ & $0.91(0.79-1.04)$ & $0.83(0.73-0.94)$ & 0.006 \\
\hline Model $2^{\varepsilon}$ & 1.00 (ref.) & $0.98(0.85-1.12)$ & $0.95(0.82-1.09)$ & $0.90(0.79-1.02)$ & 0.121 \\
\hline Model $3^{\zeta}$ & 1.00 (ref.) & $0.99(0.86-1.13)$ & $0.96(0.83-1.10)$ & $0.92(0.81-1.05)$ & 0.237 \\
\hline \multicolumn{6}{|l|}{ CVD mortality } \\
\hline No. of death & 268 & 232 & 236 & 312 & \\
\hline Model $1^{\delta}$ & 1.00 (ref.) & $0.86(0.72-1.02)$ & $0.96(0.80-1.14)$ & $0.85(0.72-1.00)$ & 0.339 \\
\hline $\operatorname{Model} 2^{\varepsilon}$ & 1.00 (ref.) & $0.88(0.74-1.05)$ & $0.99(0.83-1.18)$ & $0.93(0.79-1.10)$ & 0.978 \\
\hline Model $3^{\zeta}$ & 1.00 (ref.) & $0.88(0.74-1.05)$ & $0.99(0.83-1.18)$ & $0.94(0.79-1.11)$ & 0.972 \\
\hline \multicolumn{6}{|l|}{ Women } \\
\hline Person-years & 98,927 & 93,883 & $1,01,276$ & $1,02,466$ & \\
\hline \multicolumn{6}{|c|}{ All-cause mortality } \\
\hline No. of death & 659 & 518 & 731 & 614 & \\
\hline Model $1^{\delta}$ & 1.00 (ref.) & $0.93(0.83-1.05)$ & $1.00(0.90-1.11)$ & $0.94(0.84-1.05)$ & 0.644 \\
\hline Model $2^{\varepsilon}$ & 1.00 (ref.) & $0.97(0.87-1.09)$ & $1.04(0.94-1.16)$ & $0.98(0.88-1.10)$ & 0.722 \\
\hline Model $3^{\zeta}$ & 1.00 (ref.) & $0.98(0.87-1.10)$ & $1.05(0.94-1.17)$ & $1.00(0.89-1.12)$ & 0.574 \\
\hline \multicolumn{6}{|c|}{ Cancer mortality } \\
\hline No. of death & 228 & 161 & 235 & 215 & \\
\hline Model $1^{\delta}$ & 1.00 (ref.) & $0.82(0.67-1.01)$ & $0.94(0.79-1.13)$ & $0.94(0.78-1.14)$ & 0.723 \\
\hline Model $2^{\varepsilon}$ & 1.00 (ref.) & $0.85(0.69-1.04)$ & $0.97(0.80-1.16)$ & $0.98(0.81-1.18)$ & 0.970 \\
\hline Model $3^{\zeta}$ & 1.00 (ref.) & $0.85(0.69-1.04)$ & $0.98(0.81-1.17)$ & $0.99(0.82-1.21)$ & 0.899 \\
\hline \multicolumn{6}{|l|}{ CVD mortality } \\
\hline No. of death & 170 & 133 & 192 & 150 & \\
\hline Model $1^{\delta}$ & 1.00 (ref.) & $0.96(0.77-1.21)$ & $1.00(0.81-1.23)$ & $0.90(0.72-1.12)$ & 0.586 \\
\hline Model $2^{\varepsilon}$ & 1.00 (ref.) & $1.01(0.81-1.27)$ & $1.05(0.86-1.30)$ & $0.94(0.75-1.17)$ & 0.917 \\
\hline Model $3^{\zeta}$ & 1.00 (ref.) & $1.03(0.82-1.30)$ & $1.06(0.86-1.31)$ & $0.95(0.76-1.20)$ & 0.976 \\
\hline
\end{tabular}

${ }^{\alpha}$ Hazard ratios (HRs) and 95\% confidence intervals (95\% CIs) were calculated by Cox proportional hazards models

${ }^{\beta}$ Ranges for the quartiles of total dairy intake were 0-40.8 g/day, 44.9-109.8 g/day, 110.8-210.0 g/day, and $211.0-325.0 \mathrm{~g} /$ day in men and 0-49.4 g/day, 49.9-205.0 g/day, 210.0-224.5 g/day, and 229.3-310.0 g/day in women

${ }^{\gamma} P$ trend was calculated using the median value of each category of total dairy intake

${ }^{\delta}$ Model 1 was adjusted for age (continuous)

${ }^{\varepsilon}$ Model 2 was adjusted for Model 1 plus education level (junior high school or lower, high school, college or higher, or missing), BMI $\left(<18.5 \mathrm{~kg} / \mathrm{m}^{2}, 18.5-24.9 \mathrm{~kg} / \mathrm{m}^{2}, \geq 25.0 \mathrm{~kg} / \mathrm{m}^{2}\right.$, or missing), smoking status (never, former, $<20$ cigarettes/day, $\geq 20$ cigarettes/day, or missing), alcohol drinking status (current, never, former, or missing), history of hypertension (yes, or no), and history of diabetes (yes, or no)

${ }^{\zeta}$ Model 3 was adjusted for Model 2 plus energy intake (sex-specific tertiles or missing), fish intake (sexspecific tertiles or missing), and vegetable and fruit intake (sex-specific tertiles or missing) 
Table 3 Association between milk intake and mortality $(n=34,161)^{\alpha}$
Milk intake frequency

$P$ trend $^{\beta}$

Almost 1-2 times/week 3-4 times/week Almost daily

never/1-2

times/mo

Men

$\begin{array}{lllll}\text { Person-years } & 86,587 & 55,599 & 57,796 & 1,53,481\end{array}$

All-cause mortality

$\begin{array}{lllll}\text { No. of death } & 1131 & 635 & 632 & 1956\end{array}$

$\begin{array}{llllll}\text { Model } 1^{\gamma} & 1.00 \text { (ref.) } & 0.94(0.85-1.04) & 0.88(0.80-0.97) & 0.90(0.84-0.97) & 0.004\end{array}$

$\begin{array}{llllll}\text { Model } 2^{\delta} & 1.00 \text { (ref.) } & 0.96(0.87-1.06) & 0.92(0.83-1.01) & 0.95(0.88-1.02) & 0.184\end{array}$

$\begin{array}{llllll}\text { Model }^{\varepsilon} & 1.00 \text { (ref.) } & 0.97(0.88-1.06) & 0.93(0.84-1.02) & 0.96(0.89-1.04) & 0.339\end{array}$

Cancer mortality

No. of death

Model $1^{\gamma}$

451

Model $2^{\delta}$

1.00 (ref.)

255

1.00 (ref.)

$0.95(0.81-1.11)$

274

733

Model $2^{\circ}$

1.00 (ref.)

$0.97(0.83-1.13)$

$0.95(0.82-1.11)$

$0.85(0.75-0.95) \quad 0.005$

$1.00(0.86-1.16) \quad 0.91(0.80-1.02) \quad 0.107$

CVD mortality

No. of death

Model $1^{\gamma}$

271

1.00 (ref.)

$0.98(0.84-1.14)$

$1.01(0.87-1.18)$

$0.93(0.82-1.04) \quad 0.214$

Model $2^{\delta}$

1.00 (ref.)

139

$1.00($ ref.)

$0.86(0.71-1.06)$

150

488

Model $3^{\varepsilon}$

$0.89(0.72-1.09)$

$0.87(0.72-1.07)$

$0.93(0.80-1.08)$

0.504

$0.92(0.75-1.12)$

$1.00(0.86-1.16)$

0.794

Women

Person-years

77,460

$0.89(0.72-1.09)$

$0.92(0.76-1.13)$

$1.00(0.86-1.17)$

0.787

All-cause mortality

No. of death

527

51,494

63,856

$2,03,743$

Model $1^{\gamma}$

1.00 (ref.)

295

355

1345

Model $2^{\delta}$

1.00 (ref.)

$0.99(0.86-1.14)$

$0.92(0.80-1.05)$

$1.00(0.87-1.16)$

$0.96(0.84-1.10)$

$0.97(0.88-1.08)$

0.572

Model $3^{\varepsilon}$

1.00 (ref.)

$1.00(0.87-1.16)$

$0.97(0.84-1.11)$

$1.01(0.92-1.12)$

0.785

Cancer mortality

No. of death

183

Model $1^{\gamma}$

Model $2^{\delta}$

1.00 (ref.)

96

110

$1.02(0.92-1.14)$

0.633

Model $3^{\varepsilon}$

1.00 (ref.)

$0.90(0.70-1.15)$

$0.80(0.63-1.02)$

450

CVD mortality

No. of death

1.00 (ref.)

$0.91(0.71-1.17)$

$0.83(0.65-1.05)$

$0.94(0.79-1.11)$

0.574

$0.92(0.72-1.18)$

$0.84(0.66-1.06)$

$0.96(0.81-1.15) \quad 0.822$

$0.98(0.82-1.17) \quad 0.950$

Model $1^{\gamma}$

132

78

93

342

Model $2^{\delta}$

1.00 (ref.)

$1.09(0.82-1.44)$

$0.99(0.76-1.29)$

$0.99(0.81-1.21)$

0.732

1.00 (ref.)

$1.10(0.83-1.46)$

$1.06(0.81-1.38)$

$1.04(0.84-1.27)$

0.888

Model $3^{\varepsilon}$

1.00 (ref.)

$1.11(0.84-1.47)$

$1.08(0.82-1.41)$

$1.05(0.85-1.30)$

0.771

${ }^{\alpha}$ Hazard ratios (HRs) and $95 \%$ confidence intervals (95\% CIs) were calculated by Cox proportional hazards models

${ }^{\beta} P$ trend was calculated by treating exposure as a continuous variable

${ }^{\gamma}$ Model 1 was adjusted for age (continuous)

${ }^{8}$ Model 2 was adjusted for Model 1 plus education level (junior high school or lower, high school, college or higher, or missing), BMI ( $<18.5 \mathrm{~kg} / \mathrm{m}^{2}, 18.5-24.9 \mathrm{~kg} / \mathrm{m}^{2}, \geq 25.0 \mathrm{~kg} / \mathrm{m}^{2}$, or missing), smoking status (never, former, $<20$ cigarettes/day, $\geq 20$ cigarettes/day, or missing), alcohol drinking status (current, never, former, or missing), history of hypertension (yes, or no), and history of diabetes (yes, or no)

${ }^{\varepsilon}$ Model 3 was adjusted for Model 2 plus energy intake (sex-specific tertiles or missing), protein intake (sexspecific tertiles or missing), fish intake (sex-specific tertiles or missing), and vegetable and fruit intake (sexspecific tertiles or missing) 
Table 4 Association between yogurt intake and mortality $(n=34,161)^{\alpha}$

\begin{tabular}{|c|c|c|c|c|c|}
\hline & \multicolumn{4}{|c|}{ Yogurt intake frequency } & \multirow[t]{2}{*}{$P$ trend $^{\beta}$} \\
\hline & Almost never & $1-2$ times/mo & 1-2 times/wk & $\begin{array}{l}3 \text { times/week/ } \\
\text { Almost daily }\end{array}$ & \\
\hline \multicolumn{6}{|l|}{ Men } \\
\hline Person-years & $2,02,265$ & 82,965 & 43,393 & 24,840 & \\
\hline \multicolumn{6}{|c|}{ All-cause mortality } \\
\hline No. of death & 2680 & 883 & 458 & 333 & \\
\hline Model $1^{\gamma}$ & 1.00 (ref.) & $0.85(0.79-0.92)$ & $0.83(0.75-0.92)$ & $0.95(0.85-1.07)$ & 0.001 \\
\hline Model $2^{\delta}$ & 1.00 (ref.) & $0.90(0.83-0.97)$ & $0.88(0.80-0.97)$ & $1.02(0.91-1.14)$ & 0.111 \\
\hline Model $3^{\varepsilon}$ & 1.00 (ref.) & $0.91(0.84-0.98)$ & $0.90(0.81-0.99)$ & $1.04(0.92-1.17)$ & 0.253 \\
\hline \multicolumn{6}{|c|}{ Cancer mortality } \\
\hline No. of death & 1048 & 348 & 191 & 126 & \\
\hline Model $1^{\gamma}$ & 1.00 (ref.) & $0.86(0.76-0.97)$ & $0.89(0.76-1.04)$ & $0.92(0.77-1.11)$ & 0.064 \\
\hline Model $2^{\delta}$ & 1.00 (ref.) & $0.90(0.79-1.01)$ & $0.95(0.81-1.11)$ & $1.00(0.83-1.20)$ & 0.473 \\
\hline Model $3^{\varepsilon}$ & 1.00 (ref.) & $0.91(0.80-1.03)$ & $0.97(0.83-1.14)$ & $1.03(0.85-1.24)$ & 0.791 \\
\hline \multicolumn{6}{|l|}{ CVD mortality } \\
\hline No. of death & 647 & 213 & 112 & 76 & \\
\hline Model $1^{\gamma}$ & 1.00 (ref.) & $0.86(0.73-1.00)$ & $0.85(0.69-1.04)$ & $0.90(0.71-1.14)$ & 0.064 \\
\hline Model $2^{\delta}$ & 1.00 (ref.) & $0.92(0.79-1.08)$ & $0.91(0.74-1.11)$ & $0.99(0.78-1.25)$ & 0.448 \\
\hline Model $3^{\varepsilon}$ & 1.00 (ref.) & $0.93(0.80-1.09)$ & $0.91(0.75-1.12)$ & $0.99(0.78-1.26)$ & 0.488 \\
\hline \multicolumn{6}{|l|}{ Women } \\
\hline Person-years & $1,30,658$ & $1,06,634$ & 94,770 & 64,491 & \\
\hline \multicolumn{6}{|c|}{ All-cause mortality } \\
\hline No. of death & 987 & 612 & 538 & 385 & \\
\hline Model $1^{\gamma}$ & 1.00 (ref.) & $0.89(0.80-0.98)$ & $0.91(0.82-1.01)$ & $0.88(0.78-0.99)$ & 0.027 \\
\hline Model $2^{\delta}$ & 1.00 (ref.) & $0.91(0.82-1.01)$ & $0.94(0.84-1.04)$ & $0.91(0.81-1.03)$ & 0.109 \\
\hline Model $3^{\varepsilon}$ & 1.00 (ref.) & $0.91(0.83-1.01)$ & $0.94(0.85-1.05)$ & $0.92(0.81-1.03)$ & 0.146 \\
\hline \multicolumn{6}{|c|}{ Cancer mortality } \\
\hline No. of death & 307 & 209 & 177 & 146 & \\
\hline Model $1^{\gamma}$ & 1.00 (ref.) & $0.95(0.80-1.13)$ & $0.94(0.78-1.13)$ & $1.06(0.87-1.29)$ & 0.825 \\
\hline Model $2^{\delta}$ & 1.00 (ref.) & $0.9780 .82-1.16)$ & $0.96(0.80-1.16)$ & $1.08(0.88-1.32)$ & 0.641 \\
\hline Model $3^{\varepsilon}$ & 1.00 (ref.) & $0.98(0.82-1.17)$ & $0.97(0.81-1.18)$ & $1.10(0.89-1.34)$ & 0.541 \\
\hline \multicolumn{6}{|l|}{ CVD mortality } \\
\hline No. of death & 262 & 157 & 131 & 95 & \\
\hline Model $1^{\gamma}$ & 1.00 (ref.) & $0.89(0.73-1.08)$ & $0.88(0.71-1.08)$ & $0.84(0.67-1.07)$ & 0.110 \\
\hline Model $2^{\delta}$ & 1.00 (ref.) & $0.92(0.75-1.12)$ & $0.89(0.72-1.10)$ & $0.86(0.68-1.09)$ & 0.160 \\
\hline Model $3^{\varepsilon}$ & 1.00 (ref.) & $0.93(0.76-1.14)$ & $0.91(0.73-1.12)$ & $0.87(0.69-1.11)$ & 0.221 \\
\hline
\end{tabular}

${ }^{\alpha}$ Hazard ratios (HRs) and 95\% confidence intervals (95\% CIs) were calculated by Cox proportional hazards models

${ }^{\beta} P$ trend was calculated by treating exposure as a continuous variable

${ }^{\gamma}$ Model 1 was adjusted for age (continuous)

${ }^{8}$ Model 2 was adjusted for Model 1 plus education level (junior high school or lower, high school, college or higher, or missing), BMI $\left(<18.5 \mathrm{~kg} / \mathrm{m}^{2}, 18.5-24.9 \mathrm{~kg} / \mathrm{m}^{2}, \geq 25.0 \mathrm{~kg} / \mathrm{m}^{2}\right.$, or missing), smoking status (never, former, $<20$ cigarettes/day, $\geq 20$ cigarettes/day, or missing), alcohol drinking status (current, never, former, or missing), history of hypertension (yes, or no), and history of diabetes (yes, or no)

${ }^{\varepsilon}$ Model 3 was adjusted for Model 2 plus energy intake (sex-specific tertiles or missing), fish intake (sexspecific tertiles or missing), and vegetable and fruit intake (sex-specific tertiles or missing)

of all-cause mortality compared to non-consumers, but they only included a small number of study participants [47]. For cancer mortality, one meta-analysis reported a null association with milk intake [24], which was also suggested in an Iranian study [44]. The Japanese study found that people drinking milk had a lower risk of cancer mortality only among men aged 65 years or older, but the association was not linear [27]. In contrast, the study from China reported 
Table 5 Association between cheese intake and mortality $(n=34,161)^{\alpha}$

\begin{tabular}{|c|c|c|c|c|c|}
\hline & \multicolumn{4}{|c|}{ Cheese intake frequency } & \multirow[t]{2}{*}{$P$ trend } \\
\hline & Almost never & $1-2$ times/mo & $1-2$ times/wk & $\begin{array}{l}3 \text { times/wk/ } \\
\text { Almost daily }\end{array}$ & \\
\hline \multicolumn{6}{|l|}{ Men } \\
\hline Person-years & $1,67,125$ & $1,25,487$ & 45,575 & 15,276 & \\
\hline \multicolumn{6}{|c|}{ All-cause mortality } \\
\hline No. of death & 2276 & 1345 & 523 & 210 & \\
\hline Model $1^{\gamma}$ & 1.00 (ref.) & $0.87(0.81-0.93)$ & $0.90(0.82-1.00)$ & $0.98(0.85-1.13)$ & 0.018 \\
\hline $\operatorname{Model} 2^{\delta}$ & 1.00 (ref.) & $0.89(0.83-0.95)$ & $0.94(0.85-1.03)$ & $1.03(0.89-1.18)$ & 0.158 \\
\hline Model $3^{\varepsilon}$ & 1.00 (ref.) & $0.89(0.83-0.96)$ & $0.96(0.87-1.05)$ & $1.05(0.91-1.22)$ & 0.356 \\
\hline \multicolumn{6}{|c|}{ Cancer mortality } \\
\hline No. of death & 838 & 566 & 231 & 78 & \\
\hline Model $1^{\gamma}$ & 1.00 (ref.) & $0.99(0.89-1.11)$ & $1.09(0.94-1.26)$ & $0.99(0.79-1.25)$ & 0.548 \\
\hline Model $2^{\delta}$ & 1.00 (ref.) & $1.00(0.90-1.12)$ & $1.11(0.96-1.29)$ & $1.03(0.81-1.30)$ & 0.322 \\
\hline Model $3^{\varepsilon}$ & 1.00 (ref.) & $1.01(0.91-1.13)$ & $1.15(0.99-1.33)$ & $1.08(0.85-1.36)$ & 0.140 \\
\hline \multicolumn{6}{|l|}{ CVD mortality } \\
\hline No. of death & 573 & 309 & 115 & 51 & \\
\hline Model $1^{\gamma}$ & 1.00 (ref.) & $0.80(0.70-0.92)$ & $0.79(0.65-0.97)$ & $0.95(0.71-1.26)$ & 0.017 \\
\hline $\operatorname{Model} 2^{\delta}$ & 1.00 (ref.) & $0.82(0.72-0.95)$ & $0.86(0.70-1.05)$ & $1.00(0.75-1.34)$ & 0.114 \\
\hline Model $3^{\varepsilon}$ & 1.00 (ref.) & $0.83(0.72-0.95)$ & $0.86(0.70-1.06)$ & $1.01(0.75-1.34)$ & 0.136 \\
\hline \multicolumn{6}{|l|}{ Women } \\
\hline Person-years & $1,89,818$ & $1,24,799$ & 60,612 & 21,323 & \\
\hline \multicolumn{6}{|c|}{ All-cause mortality } \\
\hline No. of death & 1385 & 685 & 324 & 128 & \\
\hline Model $1^{\gamma}$ & 1.00 (ref.) & $0.86(0.79-0.94)$ & $0.84(0.75-0.95)$ & $0.85(0.71-1.02)$ & 0.001 \\
\hline Model $2^{\delta}$ & 1.00 (ref.) & $0.89(0.81-0.98)$ & $0.88(0.78-0.99)$ & $0.89(0.74-1.07)$ & 0.013 \\
\hline Model $3^{\varepsilon}$ & 1.00 (ref.) & $0.89(0.81-0.98)$ & $0.88(0.78-1.00)$ & $0.89(0.74-1.07)$ & 0.016 \\
\hline \multicolumn{6}{|c|}{ Cancer mortality } \\
\hline No. of death & 437 & 236 & 122 & 44 & \\
\hline Model $1^{\gamma}$ & 1.00 (ref.) & $0.92(0.79-1.08)$ & $0.98(0.80-1.20)$ & $0.92(0.67-1.25)$ & 0.536 \\
\hline Model $2^{\delta}$ & 1.00 (ref.) & $0.95(0.81-1.11)$ & $1.02(0.83-1.25)$ & $0.95(0.70-1.30)$ & 0.819 \\
\hline Model $3^{\varepsilon}$ & 1.00 (ref.) & $0.96(0.81-1.12)$ & $1.03(0.84-1.27)$ & $0.95(0.70-1.31)$ & 0.923 \\
\hline \multicolumn{6}{|l|}{ CVD mortality } \\
\hline No. of death & 354 & 172 & 83 & 36 & \\
\hline Model $1^{\gamma}$ & 1.00 (ref.) & $0.88(0.73-1.05)$ & $0.88(0.69-1.12)$ & $0.94(0.67-1.33)$ & 0.254 \\
\hline Model $2^{\delta}$ & 1.00 (ref.) & $0.90(0.75-1.09)$ & $0.93(0.73-1.18)$ & $0.99(0.70-1.40)$ & 0.546 \\
\hline Model $3^{\varepsilon}$ & 1.00 (ref.) & $0.91(0.76-1.10)$ & $0.95(0.74-1.21)$ & $0.99(0.70-1.41)$ & 0.634 \\
\hline
\end{tabular}

${ }^{\alpha}$ Hazard ratios (HRs) and 95\% confidence intervals (95\% CIs) were calculated by Cox proportional hazards models

${ }^{\beta} P$ trend was calculated by treating exposure as a continuous variable

${ }^{\gamma}$ Model 1 was adjusted for age (continuous)

${ }^{\delta}$ Model 2 was adjusted for Model 1 plus education level (junior high school or lower, high school, college or higher, or missing), BMI $\left(<18.5 \mathrm{~kg} / \mathrm{m}^{2}, 18.5-24.9 \mathrm{~kg} / \mathrm{m}^{2}, \geq 25.0 \mathrm{~kg} / \mathrm{m}^{2}\right.$, or missing), smoking status (never, former, $<20$ cigarettes/day, $\geq 20$ cigarettes/day, or missing), alcohol drinking status (current, never, former, or missing), history of hypertension (yes, or no), and history of diabetes (yes, or no)

${ }^{\varepsilon}$ Model 3 was adjusted for Model 2 plus energy intake (sex-specific tertiles or missing), fish intake (sexspecific tertiles or missing), and vegetable and fruit intake (sex-specific tertiles or missing)

that high milk consumption ( $>3$ servings/week; 1 serving $=250 \mathrm{ml}$ ) was associated with a higher cancer mortality risk [46], but the association between milk intake and cancer is rather complex considering the different effects on various site-specific cancers [48]. For CVD mortality, one meta-analysis suggested that there was no significant association with milk intake, but high heterogeneity was observed [21]. Several studies from Asian regions also did 
not find an increased risk of CVD mortality in people with higher milk intake [44, 46, 47]. The inconsistency in the milk-mortality association among previous studies may be attributable to different milk intake assessments (e.g., times/ week, servings/day, or g/day) and amounts of milk intake, the inclusion of different confounders in the models, various follow-up periods, or variations in age and the number of study participants.

For yogurt intake, we did not find any associations with mortality in both men and women. The present study is the first to examine the association between yogurt intake and all-cause and CVD mortality in the Japanese population. Previous meta-analyses did not find yogurt to be associated with the risk of mortality $[18,24,49]$, but one reported large heterogeneity $\left(I^{2}=65.8 \%, p=0.054\right)$ across studies included [18]. One meta-analysis presented subgroup results according to study regions (4 studies from Europe and 2 studies from Asia), and no association was found for both regions [49]. One of the two Asian studies was from Iran and reported a modest inverse association between yogurt intake (per serving/day; 1 serving: $230 \mathrm{~g}$ ) and all-cause and CVD mortality, but not cancer mortality [44], whereas the other from Japan only investigated the association with cancer mortality, and no association was found (daily vs. not daily) [25]. Several recent cohort studies did not show clear associations between yogurt intake and mortality risks in US and European adults [16, 28, 50, 51]. However, one multinational cohort study including both Western and Eastern populations suggested that yogurt consumption was inversely associated with all-cause mortality, although it was uncertain whether the observation was consistent across different regions [52].

The only modest association in the present study was observed between cheese intake and all-cause mortality in Japanese women. No other studies have examined the association between cheese intake and all-cause, cancer or CVD mortality in the Japanese population. Less evidence was available compared to other dairy products. Two metaanalyses both indicated that cheese intake had no association with all-cause mortality; one included 11 (RR for per 10 g/day: 0.99, 95\% CI 0.96-1.01) and the other included 9 (RR for per $50 \mathrm{~g} /$ day: $1.03,95 \%$ CI $0.99-1.07$ ) cohort studies $[18,53]$, but one observed significant heterogeneity $\left(I^{2}: 93.3 \%, p<0.001\right)[18]$. Several cohort studies were published recently, and three agreed that cheese intake was inversely associated with all-cause mortality [16, 44, 54], whereas two other studies suggested a null association [28, 50]. One meta-analysis examining the association between cheese intake and cancer mortality found no association, although the number of studies included was limited [24]. As for CVD mortality, previous studies agreed that the association between cheese intake and CVD mortality was null or modestly inverse [28, 39, 40, 50, 54, 55].

\section{Possible mechanisms}

The underlying mechanism between cheese intake and allcause mortality remains unknown, but it is suggested that cheese is rich in numerous nutrients such as whey protein or vitamin $\mathrm{K} 2$. Whey protein may have beneficial effects on reducing cardiovascular risk factors, such as improving glucose levels, insulin response and lipid profile as well as lowering blood pressure and controlling body weight [56-58]. Vitamin K2, which is exclusively synthesized by bacteria and predominantly found in fermented foods like cheese, has been shown to plays an important role in preventing metabolic syndrome [59] and CVDs [60-62], although the relation between Vitamin K2 and mortality has shown inconsistency [60, 63, 64]. Moreover, prior studies found that probiotic bacteria in fermented dairy products were reported to have positive effects on immunity, inflammation, diarrhea prevention, and cardiovascular risk factors in clinical trials [3]. Fermented dairy product intake was also inversely associated with all-cause mortality [18, 38], but one study found a marginally inverse association with cheese, but not yogurt, by examining different types of fermented food in relation to all-cause mortality [18]. More speculatively, the lack of association with yogurt may be attributed to the fact that many yogurt products on the market have a certain amount of sugar added [1]. In addition, other than the isolated effect from individual nutrient, current evidence has indicated that the matrix effect of hard cheese may have benefits in reducing the amount of fat absorbed and lowering the blood cholesterol response $[65,66]$, ultimately leading to an improvement in cardiovascular health [67]. Nevertheless, the observed association between cheese intake and all-cause mortality in the present study could simply be a chance finding due to tests of multiple outcomes. It also needs caution that the correlation between dietary records and the FFQ of cheese was lower than that of milk or yogurt in the present study, which suggested a poorer validity of cheese intake possibly owing to the low intake amount in the study population. Thus, more studies are warranted to confirm the present observation.

\section{Limitation and strengths}

Our study has some strengths, including a large number of study participants, a long period of follow-up, and a high follow-up rate. Meanwhile, some limitations should also be mentioned. First, dairy intake was obtained from a selfreported FFQ, so the misclassification of exposure would be possible. Second, dairy intake was assessed only once, at baseline, but it may change over time during follow-up. Third, details of types of dairy products (e.g., high-fat or low-fat dairy products) were not obtained, and different types of dairy products may affect health diversely. Finally, 
although a considerable number of covariates were adjusted in our models, residual and unmeasured confounding may still have affected the results.

\section{Conclusion}

The results of the present study suggested that dairy intake was not associated with all-cause, cancer, and CVD mortality in Japanese adults, except for limited evidence showing a modest association between cheese intake and a lower risk of all-cause mortality in women.

Supplementary Information The online version contains supplementary material available at https://doi.org/10.1007/s00394-021-02734-6.

Acknowledgements Y.L. received financial support from the Otsuka Toshimi Scholarship on the period of 2019-2021.

Author contributions IT and AF designed and conducted research; YL analyzed data and wrote the paper; SM, YS, and IT provided constructive suggestions; YS had primary responsibility for final content. All authors read and approved the final manuscript.

Funding This work was supported by a grant-in-aid from the Ministry of Health, Labour and Welfare, Health and Labor Sciences research grants, Japan (Comprehensive Research on Cardiovascular and LifeStyle Related Diseases: 19FA2001, 20FA1002).

Availability of data and materials and code availability Data described in the manuscript, code book, and analytic code will not be made publicly available because private information of participants were included but are available from the corresponding author on reasonable request.

\section{Declarations}

Conflict of interest All authors declare that they have no conflict of interest.

Open Access This article is licensed under a Creative Commons Attribution 4.0 International License, which permits use, sharing, adaptation, distribution and reproduction in any medium or format, as long as you give appropriate credit to the original author(s) and the source, provide a link to the Creative Commons licence, and indicate if changes were made. The images or other third party material in this article are included in the article's Creative Commons licence, unless indicated otherwise in a credit line to the material. If material is not included in the article's Creative Commons licence and your intended use is not permitted by statutory regulation or exceeds the permitted use, you will need to obtain permission directly from the copyright holder. To view a copy of this licence, visit http://creativecommons.org/licenses/by/4.0/.

\section{References}

1. Cavero-Redondo I, Alvarez-Bueno C, Sotos-Prieto M, Gil A, Martinez-Vizcaino V et al (2019) Milk and dairy product consumption and risk of mortality: an overview of systematic reviews and meta-analyses. Adv Nutr 10(suppl_2):S97-S104. https://doi. org/10.1093/advances/nmy128

2. Soedamah-Muthu SS, de Goede J (2018) Dairy consumption and cardiometabolic diseases: systematic review and updated metaanalyses of prospective cohort studies. Curr Nutr Rep 7(4):171182. https://doi.org/10.1007/s13668-018-0253-y

3. Yu E, Hu FB (2018) Dairy products, dairy fatty acids, and the prevention of cardiometabolic disease: a review of recent evidence. Curr Atheroscler Rep 20(5):24. https://doi.org/10.1007/ s11883-018-0724-z

4. Kazemi A, Barati-Boldaji R, Soltani S, Mohammadipoor N, Esmaeilinezhad $Z$ et al (2021) Intake of various food groups and risk of breast cancer: a systematic review and dose-response metaanalysis of prospective studies. Adv Nutr 12(3):809-849. https:// doi.org/10.1093/advances/nmaa147

5. World Cancer Research Fund/American Insititute for Cancer Research (2018) Continuous Update Project Expert Report 2018. Meat, fish and dairy products and the risk of cancer. https://www. wcrf.org/diet-and-cancer/

6. Barrubes L, Babio N, Becerra-Tomas N, Rosique-Esteban N, Salas-Salvado J (2019) Association between dairy product consumption and colorectal cancer risk in adults: a systematic review and meta-analysis of epidemiologic Studies. Adv Nutr 10(suppl_2):S190-S211. https://doi.org/10.1093/advances/ nmy 114

7. Aljuraiban GS, Stamler J, Chan Q, Van Horn L, Daviglus ML et al (2018) Relations between dairy product intake and blood pressure: the INTERnational study on MAcro/micronutrients and blood Pressure. J Hypertens 36(10):2049-2058. https://doi.org/10. 1097/HJH.0000000000001779

8. Schmidt KA, Cromer G, Burhans MS, Kuzma JN, Hagman DK et al (2021) Impact of low-fat and full-fat dairy foods on fasting lipid profile and blood pressure: exploratory endpoints of a randomized controlled trial. Am J Clin Nutr 114(3):882-892. https:// doi.org/10.1093/ajen/nqab131

9. Rancourt-Bouchard M, Gigleux I, Guay V, Charest A, SaintGelais D et al (2020) Effects of regular-fat and low-fat dairy consumption on daytime ambulatory blood pressure and other cardiometabolic risk factors: a randomized controlled feeding trial. Am J Clin Nutr 111(1):42-51. https://doi.org/10.1093/ajen/nqz251

10. Sacks FM, Lichtenstein AH, Wu JHY, Appel LJ, Creager MA et al (2017) Dietary fats and cardiovascular disease: a presidential advisory from the American Heart Association. Circulation 136(3):e1-e23. https://doi.org/10.1161/CIR.0000000000000510

11. Huth PJ, Park KM (2012) Influence of dairy product and milk fat consumption on cardiovascular disease risk: a review of the evidence. Adv Nutr 3(3):266-285. https://doi.org/10.3945/an.112. 002030

12. Lopez-Plaza B, Bermejo LM, Santurino C, Cavero-Redondo I, Alvarez-Bueno C et al (2019) Milk and dairy product consumption and prostate cancer risk and mortality: an overview of systematic reviews and meta-analyses. Adv Nutr 10(suppl_2):S212S223. https://doi.org/10.1093/advances/nmz014

13. Harrison S, Lennon R, Holly J, Higgins JPT, Gardner M et al (2017) Does milk intake promote prostate cancer initiation or progression via effects on insulin-like growth factors (IGFs)? A systematic review and meta-analysis. Cancer Causes Control 28(6):497-528. https://doi.org/10.1007/s10552-017-0883-1

14. Ganmaa D, Cui X, Feskanich D, Hankinson SE, Willett WC (2012) Milk, dairy intake and risk of endometrial cancer: a 26-year follow-up. Int J Cancer 130(11):2664-2671. https://doi. org/10.1002/ijc. 26265

15. Willett WC, Ludwig DS (2020) Milk and health. N Engl J Med 382(7):644-654. https://doi.org/10.1056/NEJMra1903547

16. Mazidi M, Mikhailidis DP, Sattar N, Howard G, Graham I et al (2019) Consumption of dairy product and its association with total 
and cause specific mortality - a population-based cohort study and meta-analysis. Clin Nutr 38(6):2833-2845. https://doi.org/ 10.1016/j.clnu.2018.12.015

17. Schwingshackl L, Schwedhelm C, Hoffmann G, Lampousi AM, Knuppel S et al (2017) Food groups and risk of all-cause mortality: a systematic review and meta-analysis of prospective studies. Am J Clin Nutr 105(6):1462-1473. https://doi.org/10.3945/ajcn. 117.153148

18. Guo J, Astrup A, Lovegrove JA, Gijsbers L, Givens DI et al (2017) Milk and dairy consumption and risk of cardiovascular diseases and all-cause mortality: dose-response meta-analysis of prospective cohort studies. Eur J Epidemiol 32(4):269-287. https://doi. org/10.1007/s10654-017-0243-1

19. Mullie P, Pizot C, Autier P (2016) Daily milk consumption and all-cause mortality, coronary heart disease and stroke: a systematic review and meta-analysis of observational cohort studies. BMC Public Health 16(1):1236. https://doi.org/10.1186/ s12889-016-3889-9

20. Larsson SC, Crippa A, Orsini N, Wolk A, Michaelsson K (2015) Milk consumption and mortality from all causes, cardiovascular disease, and cancer: a systematic review and meta-analysis. Nutrients 7(9):7749-7763. https://doi.org/10.3390/nu7095363

21. O'Sullivan TA, Hafekost K, Mitrou F, Lawrence D (2013) Food sources of saturated fat and the association with mortality: a meta-analysis. Am J Public Health 103(9):e31-42. https://doi. org/10.2105/AJPH.2013.301492

22. Soedamah-Muthu SS, Ding EL, Al-Delaimy WK, Hu FB, Engberink MF et al (2011) Milk and dairy consumption and incidence of cardiovascular diseases and all-cause mortality: dose-response meta-analysis of prospective cohort studies. Am J Clin Nutr 93(1):158-171. https://doi.org/10.3945/ajen.2010. 29866

23. Elwood PC, Pickering JE, Givens DI, Gallacher JE (2010) The consumption of milk and dairy foods and the incidence of vascular disease and diabetes: an overview of the evidence. Lipids 45(10):925-939. https://doi.org/10.1007/s11745-010-3412-5

24. Lu W, Chen H, Niu Y, Wu H, Xia D et al (2016) Dairy products intake and cancer mortality risk: a meta-analysis of 11 populationbased cohort studies. Nutr J 15(1):91. https://doi.org/10.1186/ s12937-016-0210-9

25. Matsumoto M, Ishikawa S, Nakamura Y, Kayaba K, Kajii E (2007) Consumption of dairy products and cancer risks. J Epidemiol 17(2):38-44. https://doi.org/10.2188/jea.17.38

26. Kondo I, Ojima T, Nakamura M, Hayasaka S, Hozawa A et al (2013) Consumption of dairy products and death from cardiovascular disease in the Japanese general population: the NIPPON DATA80. J Epidemiol 23(1):47-54. https://doi.org/10.2188/jea. je20120054

27. Wang C, Yatsuya H, Tamakoshi K, Iso H, Tamakoshi A (2015) Milk drinking and mortality: findings from the Japan collaborative cohort study. J Epidemiol 25(1):66-73. https://doi.org/10.2188/ jea.JE20140081

28. Ding M, Li J, Qi L, Ellervik C, Zhang X et al (2019) Associations of dairy intake with risk of mortality in women and men: three prospective cohort studies. BMJ. https://doi.org/10.1136/ bmj.16204

29. Tognon G, Nilsson LM, Shungin D, Lissner L, Jansson JH et al (2017) Nonfermented milk and other dairy products: associations with all-cause mortality. Am J Clin Nutr 105(6):1502-1511. https://doi.org/10.3945/ajcn.116.140798

30. Fukao A, Tsubono Y, Komatsu S, Tsuji I, Minami Y et al (1995) A cohort study on the relation of lifestyle, personality and biologic markers to cancer in Miyagi, Japan: study design, response rate and profiles of the cohort subjects. J Epidemiol 5(3):153-157

31. Tsuji I, Nishino Y, Ohkubo T, Kuwahara A, Ogawa K et al (1998) A prospective cohort study on National Health Insurance beneficiaries in Ohsaki, Miyagi Prefecture, Japan: study design, profiles of the subjects and medical cost during the first year. J Epidemiol 8(5):258-263. https://doi.org/10.2188/jea.8.258

32. Ogawa K, Tsubono Y, Nishino Y, Watanabe Y, Ohkubo T et al (2003) Validation of a food-frequency questionnaire for cohort studies in rural Japan. Public Health Nutr 6(2):147-157. https:// doi.org/10.1079/PHN2002411

33. Science and Technology Agency (1982) Standard Tables of Food Composition in Japan, 4th edn. Printing Bureau, Ministry of Finance, Tokyo

34. Nakaya N, Tsubono Y, Hosokawa T, Nishino Y, Ohkubo T et al (2003) Personality and the risk of cancer. J Natl Cancer Inst 95(11):799-805. https://doi.org/10.1093/jnci/95.11.799

35. Nakaya N, Tsubono Y, Nishino Y, Hosokawa T, Fukudo S et al (2005) Personality and cancer survival: the Miyagi cohort study. Br J Cancer 92(11):2089-2094. https://doi.org/10.1038/sj.bjc. 6602610

36. Matsuyama S, Zhang S, Tomata Y, Abe S, Tanji F et al (2020) Association between improved adherence to the Japanese diet and incident functional disability in older people: The Ohsaki Cohort 2006 Study. Clin Nutr 39(7):2238-2245. https://doi.org/10.1016/j. clnu.2019.10.008

37. Ministry of Health, Labour and Welfare (1992) The National Health and Nutrition Survey in Japan, 1990. Daiichi Shuppan, Tokyo

38. Soedamah-Muthu SS, Masset G, Verberne L, Geleijnse JM, Brunner EJ (2013) Consumption of dairy products and associations with incident diabetes, CHD and mortality in the Whitehall II study. Br J Nutr 109(4):718-726. https://doi.org/10.1017/S0007 114512001845

39. Bonthuis M, Hughes MC, Ibiebele TI, Green AC, van der Pols JC (2010) Dairy consumption and patterns of mortality of Australian adults. Eur J Clin Nutr 64(6):569-577. https://doi.org/10.1038/ ejcn. 2010.45

40. van Aerde MA, Soedamah-Muthu SS, Geleijnse JM, Snijder MB, Nijpels G et al (2013) Dairy intake in relation to cardiovascular disease mortality and all-cause mortality: the Hoorn Study. Eur J Nutr 52(2):609-616. https://doi.org/10.1007/s00394-012-0363-z

41. Michaelsson K, Wolk A, Langenskiold S, Basu S, Warensjo Lemming E et al (2014) Milk intake and risk of mortality and fractures in women and men: cohort studies. BMJ. https://doi.org/10.1136/ bmj.g6015

42. Praagman J, Franco OH, Ikram MA, Soedamah-Muthu SS, Engberink MF et al (2015) Dairy products and the risk of stroke and coronary heart disease: the Rotterdam Study. Eur J Nutr 54(6):981-990. https://doi.org/10.1007/s00394-014-0774-0

43. Huang LY, Wahlqvist ML, Huang YC, Lee MS (2014) Optimal dairy intake is predicated on total, cardiovascular, and stroke mortalities in a Taiwanese cohort. J Am Coll Nutr 33(6):426-436. https://doi.org/10.1080/07315724.2013.875328

44. Farvid MS, Malekshah AF, Pourshams A, Poustchi H, Sepanlou SG et al (2017) dairy food intake and all-cause, cardiovascular disease, and cancer mortality: The Golestan Cohort Study. Am J Epidemiol 185(8):697-711. https://doi.org/10.1093/aje/kww139

45. Talaei M, Koh WP, Yuan JM, Pan A (2017) The association between dairy product intake and cardiovascular disease mortality in Chinese adults. Eur J Nutr 56(7):2343-2352. https://doi.org/ 10.1007/s00394-016-1274-1

46. Wang XJ, Jiang CQ, Zhang WS, Zhu F, Jin YL et al (2020) Milk consumption and risk of mortality from all-cause, cardiovascular disease and cancer in older people. Clin Nutr 39(11):3442-3451. https://doi.org/10.1016/j.clnu.2020.03.003

47. Talaei M, Hosseini N, van Dam RM, Sadeghi M, Oveisgharan $S$ et al (2019) Whole milk consumption and risk of cardiovascular disease and mortality: Isfahan Cohort Study. Eur J Nutr 58(1):163-171. https://doi.org/10.1007/s00394-017-1581-1 
48. Jeyaraman MM, Abou-Setta AM, Grant L, Farshidfar F, Copstein L et al (2019) Dairy product consumption and development of cancer: an overview of reviews. BMJ Open 9(1):e023625. https:// doi.org/10.1136/bmjopen-2018-023625

49. Gao X, Jia HY, Chen GC, Li CY, Hao M (2020) Yogurt intake reduces all-cause and cardiovascular disease mortality: a metaanalysis of eight prospective cohort studies. Chin J Integr Med 26(6):462-468. https://doi.org/10.1007/s11655-020-3085-8

50. Pala V, Sieri S, Chiodini P, Masala G, Palli D et al (2019) Associations of dairy product consumption with mortality in the European Prospective Investigation into Cancer and Nutrition (EPIC)-Italy cohort. Am J Clin Nutr 110(5):1220-1230. https://doi.org/10. 1093/ajen/nqz183

51. Schmid D, Song M, Zhang X, Willett WC, Vaidya R et al (2020) Yogurt consumption in relation to mortality from cardiovascular disease, cancer, and all causes: a prospective investigation in 2 cohorts of US women and men. Am J Clin Nutr 111(3):689-697. https://doi.org/10.1093/ajcn/nqz345

52. Dehghan M, Mente A, Rangarajan S, Sheridan P, Mohan V et al (2018) Association of dairy intake with cardiovascular disease and mortality in 21 countries from five continents (PURE): a prospective cohort study. Lancet 392(10161):2288-2297. https://doi.org/ 10.1016/S0140-6736(18)31812-9

53. Tong X, Chen GC, Zhang Z, Wei YL, Xu JY et al (2017) Cheese consumption and risk of all-cause mortality: a meta-analysis of prospective studies. Nutrients. https://doi.org/10.3390/nu9010063

54. Tognon G, Rothenberg E, Petrolo M, Sundh V, Lissner L (2018) Dairy product intake and mortality in a cohort of 70-year-old Swedes: a contribution to the Nordic diet discussion. Eur J Nutr 57(8):2869-2876. https://doi.org/10.1007/s00394-017-1556-2

55. Praagman J, Dalmeijer GW, van der Schouw YT, SoedamahMuthu SS, Monique Verschuren WM et al (2015) The relationship between fermented food intake and mortality risk in the European Prospective Investigation into Cancer and Nutrition-Netherlands cohort. Br J Nutr 113(3):498-506. https://doi.org/10.1017/S0007 114514003766

56. Pal S, Radavelli-Bagatini S (2013) The effects of whey protein on cardiometabolic risk factors. Obesity Rev 14(4):324-343. https:// doi.org/10.1111/obr.12005

57. Bjornshave A, Hermansen K (2014) Effects of dairy protein and fat on the metabolic syndrome and type 2 diabetes. Rev Diabet Stud 11(2):153-166. https://doi.org/10.1900/RDS.2014.11.153

58. Sousa GT, Lira FS, Rosa JC, de Oliveira EP, Oyama LM et al (2012) Dietary whey protein lessens several risk factors for metabolic diseases: a review. Lipids Health Dis. https://doi.org/ 10.1186/1476-511X-11-67

59. Dam V, Dalmeijer GW, Vermeer C, Drummen NE, Knapen MH et al (2015) Association between Vitamin K and the metabolic syndrome: a 10-year follow-up study in adults. J Clin Endocrinol Metab 100(6):2472-2479. https://doi.org/10.1210/jc.2014-4449

60. Chen HG, Sheng LT, Zhang YB, Cao AL, Lai YW et al (2019) Association of vitamin $\mathrm{K}$ with cardiovascular events and all-cause mortality: a systematic review and meta-analysis. Eur J Nutr 58(6):2191-2205. https://doi.org/10.1007/s00394-019-01998-3

61. Bellinge JW, Dalgaard F, Murray K, Connolly E, Blekkenhorst LC et al (2021) Vitamin K Intake and Atherosclerotic Cardiovascular Disease in the Danish Diet Cancer and Health Study. J Am Heart Assoc 10(16):e020551. https://doi.org/10.1161/JAHA.120.020551

62. Geleijnse JM, Vermeer C, Grobbee DE, Schurgers LJ, Knapen MH et al (2004) Dietary intake of menaquinone is associated with a reduced risk of coronary heart disease: the Rotterdam Study. J Nutr 134(11):3100-3105. https://doi.org/10.1093/jn/134.11.3100

63. Zwakenberg SR, den Braver NR, Engelen AIP, Feskens EJM, Vermeer C et al (2017) Vitamin K intake and all-cause and cause specific mortality. Clin Nutr 36(5):1294-1300. https://doi.org/10. 1016/j.clnu.2016.08.017

64. Nimptsch K, Rohrmann S, Kaaks R, Linseisen J (2010) Dietary vitamin $\mathrm{K}$ intake in relation to cancer incidence and mortality: results from the Heidelberg cohort of the European Prospective Investigation into Cancer and Nutrition (EPIC-Heidelberg). Am J Clin Nutr 91(5):1348-1358. https://doi.org/10.3945/ajen.2009. 28691

65. Thorning TK, Bertram HC, Bonjour JP, de Groot L, Dupont D et al (2017) Whole dairy matrix or single nutrients in assessment of health effects: current evidence and knowledge gaps. Am J Clin Nutr 105(5):1033-1045. https://doi.org/10.3945/ajen.116.151548

66. Feeney EL, Barron R, Dible V, Hamilton Z, Power Y et al (2018) Dairy matrix effects: response to consumption of dairy fat differs when eaten within the cheese matrix-a randomized controlled trial. Am J Clin Nutr 108(4):667-674. https://doi.org/10.1093/ ajcn/nqy 146

67. Feeney EL, Lamichhane P, Sheehan JJ (2021) The cheese matrix: Understanding the impact of cheese structure on aspects of cardiovascular health - a food science and a human nutrition perspective. Int J Dairy Technol. https://doi.org/10.1111/1471-0307. 12755 